Vol. 3, No. 2 | July - December 2020

\title{
Computation of Electronic Conductivity of Pentance As Semiconductor Material Using Castep Simulations
}

\author{
Nasif Raza Jaffri ${ }^{1}$, Liu Shi ${ }^{1}$, Aftab Ahmad Rao ${ }^{2}$, Usama Abrar ${ }^{1}$, and Jian \\ Yang ${ }^{1}$
}

\begin{abstract}
:
Density function theory (DFT) interprets the stochastic behavior of electrons and hence predicts solid-state properties of materials. CASTEP; a software to compute charge densities, spin densities, wavefunctions, relaxed atomic positions, forces, the Fermi lev-el, the total energy, and symmetry operations. Conventionally, inorganic materials like $\mathrm{Ge}, \mathrm{Si}$ used to produce electronic devices. Lately, organic electronics are introduced and is a growing branch of the electronics industry. Although the rate of growth of this industry is high still, this industry is in its early stages. An organic field-effect transistor (OFET) is the major unit cells of this industry. To use organic materials as OFETs; semiconductor material bandgap must be known. In this work; the CASTEP software package was used to calculate Pentacene (organic material) bandgap, bandgap types, and values. It came up with the results that Pentacene is a material with direct bandgap and good electron conductivities. These instructions give basic guidelines for preparing camera-ready papers.
\end{abstract}

Keywords: Organic Electronics, OFET, CASTEP, PENTACENE, DFT.

\section{Introduction}

The term organic semiconductors refer to organic materials with characteristics similar to inorganic semiconductors (e.g. $\mathrm{Si}, \mathrm{Ge})$. Like inorganic semiconductors, holes and electrons are responsible for the flow of current in organic semiconductors [1]. But the difference between these organic and inorganic semiconductors is that organic semiconductors have $\pi$ conjugate bonds which allow electron movements through $\pi$ electron cloud overlap. However, current flow through inorganic semiconductors is due to electron jumping between localized states and mobility gaps [2].

The history of solid-state electronic devices is not long. The first remarkable product produced in the electronics industry was a transistor in the year 1947. In a couple of decades, transistors were readily available at reasonable prices. Transistors can be seen nearly in every type of electronic circuitry (e.g. from laptops to children's toys). Even nowadays many types of displays also use transistors (TFTs, LEDs). These displays are replacing older technology (CRT's) quickly because they are cheap, cover less space and consume less power with good contrast ratio, brightness, and colours. In the last 50 years, silicon has been the major raw material in electronics manufacturing. Electronic properties of organic solids were first noticed in the 1960s. At that time, only a basic understanding of the materials was known, by mainly focusing on one

\footnotetext{
${ }^{1}$ School of Control and Computer Engineering. North China Electric Power University Beijing, 102206 China

${ }^{2}$ Department of Electrical Engineering, NFC-Institute of Engineering and Fertilizer Research Faisalabad, Pakistan.

Corresponding Author: nasifraza@hotmail.com
} 
component of organic molecular crystals. However, it was soon concluded that like inorganic materials, roots of electrons and holes could be defined for organic materials as the charge carrier band is narrow in this case [3].

The story of organic materials started way back in 1862, when Henry Letheby of college London Hospital observed conductivity in organic material, probably polyaniline [4]. In 1970 conductivity of organic polymers at low temperature was noted. Alan J. Heeger, Alan G. MacDiarmid, and Hideki Shirakawa are often credited for the "discovery and development" of conductive polymers and were jointly awarded the Nobel Prize in Chemistry in 2000 for their 1977 report of similarly-oxidized and iodine-doped polyacetylene [5].

Organic materials composed of primarily covalent bonds because of their structure, organic materials can either act as semiconductors or insulators [5]. Organic electronics were first spotted for commercial interest when their photoconductive properties were observed. One of the problems with organic materials is their instability especially as they can degrade after exposure to air, water or light. So, there are a few limitations regarding the employment of organic structure and their manipulation. As electrons are primarily in covalent bonds, the electron mobility in organic materials is nowhere near their mobility in semiconductor materials [6].

Organic devices efficiency depends upon the charge injection and chances of charge capture or from the balance of injection of electrons one side and holes from another side [7]. The device is in between two electrodes the negative is called lowest unoccupied molecular orbital (LUMO) and positive as highest occupied Molecular orbital (HOMO).

\section{OFETs}

Organic field effect transistors (OFETS) shows many advantages. Their ability to deposit on the flexible substrate made it a popular product of organic electronics [8].
Insulator, semiconductor, and electrodes are basic components of FET. In case of organic electronics, the semiconductor is composed of some organic material, while inorganic material can be used for others [1]. But some manufacturers prefer to make every part by using organic material. These devices are used on the flexible substrate for high switching applications [9] [10].
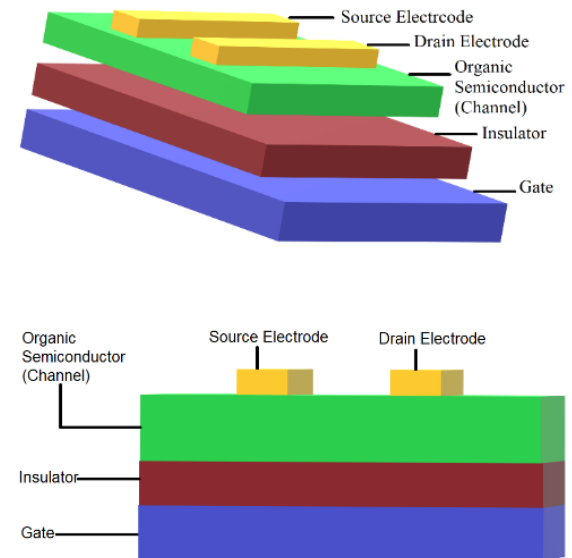

Fig 1. Layer by layer structure of OFET

Oligomers and polymers are two elementary segregations of organic semiconductors. Oligomers are small molecules that are usually prepared using vacuum processes, and polymers with long chains are prepared using wet chemical techniques. Despite, the process of preparation or type of material, the basic intension behind these materials is an efficient transfer charge carrier (electron or hole) [11]. The efficiency of materials is sometimes measured by the ease of charge injection in the material using electrodes. Normally, the material is known as a good charge transporter when ionization energy is closer to the Fermi level of electrodes [12].

In the absence of a voltage potential, the charge trans-fer is due to the diffusion which is defined by the equation.

$$
\left\langle x^{2}\right\rangle=n D t
$$

Where $\left\langle x^{2}\right\rangle$ is the displace D of charge is the diffusion coefficient, $\mathrm{t}$ is the time, $\mathrm{n}$ can be equal to 2,4 or 6 depending on 1- 
dimensional, 2-dimensional or 3dimensional movement. The relation between the charge mobility $\mu$ and diffusion coefficient $\mathrm{D}$ is defined by the equation.

$$
\mu=\frac{e D}{k_{b} T}
$$

And $\mathrm{e}$ is electron charge; $\mathrm{kb}$ is Boltzmann constant $\mathrm{T}$ for temperature.

If the external electric field (F) applied will make the charge carrier drift, the movement of the charge carrier creates a velocity (v) in the semiconductor. Charge mobility $\mu$ can also be defined with relation to field applied and velocity produced.

$$
\mu=\frac{v}{F}
$$

\section{A. Time of Flight (Tof)}

A Charge carrier which is injected from one electrode with the help of applied potential, the time is taken by a carrier to reach another electrode is known as the Time of flight (Tof). The time of flight is used to measure the structural defects of materials.

\section{As known that}

$$
v=\text { velocity }=\frac{\text { displacement }}{\text { time }}=\frac{d}{t} \quad \text { and }
$$

$F=$ Electric Field $=$

$\frac{\text { Applied Voltage }}{\text { Distance between electrodes }}$ replacing these two values in equation (3) gives

$$
\mu=\frac{d^{2}}{v t}
$$

\section{B. Time of Flight (Tof) for OFETs}

The behavior of the inorganic transistor in the linear and saturated region is approximately the same as it for organic materials [12]. This means the source to drain current equations for both regions of inorganic material FET is also applicable for OFET [11].

The equation for source unsaturated region is

$$
I_{S D}=\frac{W}{L} \mu C\left(V_{G}-V_{T}\right) V_{D S}
$$

And the equation for the saturated region is

$$
I_{S D}=\frac{W}{2 L} \mu C\left(V_{G}-V_{T}\right)^{2}
$$

\section{Material Under study}

Benzene, C6H6, has six carbon atoms arranged in a ring. Compounds which are formed with the combination of the benzene ring are called arenes or aromatics [13].

These compounds exhibit the properties of benzene on an extended scale. Pentacene is composed of five Benzene rings. In order to completely understand the properties of Pentacene, the basic behavior of Benzene must be known.<smiles>c1ccc2cc3cc4cc5ccccc5cc4cc3cc2c1</smiles>

Fig 2. Five benzene rings combine to form Pentacene

Benzene with chemical formula C6H6 is a colorless and flammable liquid aromatic hydro-carbon possessing a distinct, characteristic odor with volatile nature. A major use of benzene is in the preparation of other organic compounds on an industrial scale. In the past, benzene was used in the shoe and garment industries as a sol-vent and for the preparation of some medicines [14].

In semiconductors, field mobility means charge/electron mobility, which means how easy electrons transport through materials Pentacene has all of these properties and was selected for the development of OFETS or OLEDs [8].<smiles>Ic1c(I)c(I)c(I)c(I)c1I</smiles>

Fig 3. Benzene representation

\section{A. Material Properties}

Pentacene; a dark blue powder shows sensitivity to light. The organic phototransistor (PTs) shows high mobility of $\mathrm{cm} 0.42 \mathrm{~V}-1 \mathrm{~s}-1$, a maximum photo responsivity of about $1000 \mathrm{~A} / \mathrm{W}$, and photocurrent/dark-current ratio in the region of 800 under white light irradiation 
of $30 \mu \mathrm{W} / \mathrm{cm} 2$ [15]. The properties are comparable with those of PTs based on amorphous silicon. Meanwhile, it is interesting to see that the devices show a high air-stable property and high photosensitivity via the electric-field enhanced process [1]. All these phenomena were attributed to the intrinsic opticelectronic property of the organic semiconductor and optic-electric field effect.

\section{B. Mobilities in Material}

The mobility of holes in Pentacene is higher as compared to other organic materials. It tends to form well-ordered molecular crystals. Pentacene exhibit mobilities in excess of $1 \mathrm{~cm} 2 / \mathrm{Vs}$. SiO2. Transistors can exhibit mobilities in excess of $1 \mathrm{~cm} 2 / \mathrm{Vs}$ [14].

OFETs which are made up of using single-crystal Pentacene having up to 0.30 $\mathrm{cm} 2 / \mathrm{Vs}$ hole conductivity at room temperature, effective mobility, $\mu$ eff $V s$ and on/off ratios up to $5 \times 106$. The activation energy (Ea) of a transistor can be reduced to $0.143 \mathrm{eV}$ at room temperature by applying a negative gate voltage of about $-50 \mathrm{~V}$. With $\mathrm{Ea}=0.143 \mathrm{eV}[12]$, the material reached a thermal equilibrium between trapped and free carriers, and it's found that free carriers are about $0.4 \%$ of total injected carries.

In modern microelectronics, semiconductors are inject-ed with free carriers. This controlled injection is very helpful in controlling the mobility of the devices. Organic semiconductors have the advantage of flexible processing of largearea devices at low cost. FETs are used to investigate the physical properties of these materials. It's still the fact that accuracy achieved using inorganic materials is much better than the accuracy of organic materials. One of the major reasons for low accuracy is that intrinsic organic material has inter and intramolecular vibrational modes which scatter chare carrier and reduce mobility $(\mu)$. This drawback is not exhibited by inorganic materials which is the reason for their high mobility.

Polyacenes exhibit good properties for organic materials; among them, pentacene is one with the most promising features.
Among all polyacenes, pentacene has the smallest bandgap and has the highest effective mobility in polycrystalline organic thin-film FETs, $\mu$ eff $=0.3-1.5 \mathrm{~cm} 2 / \mathrm{Vs}$. the value is comparable with amorphous $\mathrm{Si}$ devices. Another important notable feature is that, as the crystalline of the device increases, $\mu$ eff also increases [11]. This fact reveals the need for a single crystal and requires the fabrication of single crystals for FETs manufacture.

A single crystal of pentacene FET was put under study, and it shows different results. The large negative $\mathrm{Vg}$ of about $50 \mathrm{~V}$, Ea (activation) energy to the value $0.143 \mathrm{eV}$. The Ea depends on EF and EV by $\mathrm{Ea} \sim \mathrm{EF}-\mathrm{EV}$ on EF is the given Fermi level of the material. Ea on $\mathrm{Vg}$ changes to the gradual shift of the hole Fermi energy toward the valence band as more empty places become filled because of FET hole injection [1]. The number of injected carriers per unit area is given by

$$
C_{S} V_{g}=\int_{E_{F 1}}^{E_{F 0}} d E N_{t}(E)
$$

$\mathrm{Nt}(\mathrm{E})$ is trapped carriers' energy distribution function of per unit area. While EF0 and EF1 are Fermi energy levels without and with Vg on, respectively. Let's assume at any injection rate thermal equilibrium developed be-tween free and trap carriers [6]. The number of thermal-ly excited free carriers is given by

$P_{f}=\int_{E_{F 1}}^{E_{C}} d E N_{t}(E) \exp \left(E_{V}-E\right) / k_{b} T(8)$

The conduction band energy Ec upper limit of the integral can be replaced with EF0 in equation (8) as they are approximately the same at room temperature. The simplified expression will be

$$
P_{f}=\exp \left(\left(E_{V}-E\right) / k_{b} T\right) \int_{E_{F 1}}^{E_{C}} d E N_{t}(E)
$$

The value of $\int_{E_{F 1}}^{E_{C}} d E N_{t}(E)$ from equation (7) can be replaced in equation (9), the new equation will be

$$
P_{f}=\exp \left(\left(E_{V}-E\right) / k_{b} T\right) C_{S} V_{g}
$$

We can see that from the graph below that at $E_{V}-E_{F 1}$ is approximately equal to activation energy Ea with negative sign - 
$0.143 \mathrm{eV}$. This gives that the free carriers are about $0.4 \%$ of the injected ones.

\section{CASTEP Simulations}

CASTEP is a software package that provides the decent atomic level description. This software is used for the prediction of material properties, e.g. energies (stress, forces), electronic structure, geometry optimization, molecular dynamic, magnetic responses, phonons, electric field responses, etc.

The basic idea used in this software is the construction of Hamiltonian, and after the construction of that Hamiltonian, it calculates the full Hamiltonian equation. To perform this task Castep requires more than one input file, there are two input files in CASTEP * cell and *. param. These two files can be open simply by using windows notepad. The *.cell file contains the position of atoms in molecules hence contains the structure which has to be calculated, while * param contains the information on how this calculation should be done. This code can be found in the appendix.

The first line of code in parameter code is a comment; the software user can write anything here for himself to make a reference; it won't affect the output. As I tried to optimize the geometry of diamond, I wrote a comment - Diamond Geometry Optimization. The second line in the parameter file defines the task which has to be performed; in this case, it is Geometry Optimization; it can be phonons, band structure, NMR or single point energy. By default, it goes for single-point energy. The third line is for the exchange-correlation function as density functional theory uses local density approximation (LDA) as its obvious choice for calculation; similarly, LDA is default functional here. But other hybrid and new functions can also be used for calculation such as PW91, LDC-X, WC, B3LYP, etc. The next line in the parameter file is optimization speed, which directly relates to the RAM of the system, the default value is 0 , but it can be increased by changing it to $+1,+2, \ldots$ or decreased by $-1,-$ $2,-3 \ldots$ fix occupancy is used to define the material as insulator or metal. The last line is the method defined for geometry optimization. It can be damped MD (damped molecular dynamics) or delocalized but BFGS is the default.

The Appendix contains the code for cell file. The block-lattice consists of lattice parameters a, b, c and angles $\alpha, \beta, \gamma$ which defines how the lattice grows. Then the position of atoms on $\mathrm{x}, \mathrm{y}, \mathrm{z}$ plane along with the $\mathrm{k}$ point. This input file is run by using plate form of MSDOS and can take from a few minutes to days for the completion depending upon the size of molecules, latticmeter, and method used for calculation

After the simulation, the software creates an output file with the name of *.cst. This file contains the desired information. The first section consists of memory requirement for the job, next control parameters, variables used, system geometry, pseudopotentials, k-points, etc. The information produces in the output file was used for the calculations which are presented in the result section.

\section{Results and Discussions}

CASTEP calculates energies by growing periodically into three directions a, $\mathrm{b}$ and $\mathrm{c}$. Wavenumber $\mathrm{k}$ is de-fined as the number of wavelengths per unit distance. Waves bombarded along each axis on the lattice and hence changes wavenumber $(\mathrm{k})$ and changing the num-ber of wavelengths affects the factor $\mathrm{k} / \mathrm{a}, \mathrm{k} / \mathrm{b}$ or $\mathrm{k} / \mathrm{c}$ along with all three directions. This factor is varied from its maximum to minimum value $(0-1)$ in many steps by software to calculate the energies of electrons involved. Then these energies were drawn factor $\mathrm{k} / \mathrm{a}, \mathrm{k} / \mathrm{b}$ and $\mathrm{k} / \mathrm{c}$ again to find bandgap. As it is discussed in chapter 4 that there are many techniques to use density function-al theory; however, in these calculations, four of them (LDA-X, PBE, PW91and WC) are used [16].

There are two types of bandgaps in semiconductors, direct bandgap, and indirect bandgap. If the conduction band minimum and valence band maximum occur at the same point in $\mathrm{k}$-space, then the bandgap is known as a direct bandgap; otherwise, it is indirect [17]. The bandgap calculated is the difference between HOMO and LUMO levels. 
Table I: Electron energies for lda-x, k/a, k/b and k/c

\begin{tabular}{|c|c|c|c|c|c|c|}
\hline \multirow{3}{*}{ ن } & $\mathrm{k} / \mathrm{a}$ & 0 & 0.27 & 0.55 & 0.73 & 1 \\
\hline & $\mathrm{k} / \mathrm{b}$ & 0 & 0.22 & 0.56 & 0.78 & 1 \\
\hline & $\mathrm{k} / \mathrm{c}$ & 0 & 0.2 & 0.6 & 0.8 & 1 \\
\hline \multirow{3}{*}{$\sum_{\substack{0 \\
0}}^{0}$} & $\mathrm{k} / \mathrm{a}$ & 0.76 & 0.78 & 0.71 & 0.78 & 0.76 \\
\hline & $\mathrm{k} / \mathrm{b}$ & 0.757 & 0.678 & 0.484 & 0.678 & 0.757 \\
\hline & $\mathrm{k} / \mathrm{c}$ & 0.757 & 0.752 & 0.743 & 0.752 & 0.757 \\
\hline \multirow{3}{*}{$\varliminf_{ٍ}^{\varrho}$} & $\mathrm{k} / \mathrm{a}$ & 1.57 & 1.57 & 1.65 & 1.57 & 1.57 \\
\hline & $\mathrm{k} / \mathrm{b}$ & 1.568 & 1.625 & 1.772 & 1.625 & 1.568 \\
\hline & $\mathrm{k} / \mathrm{c}$ & 1.568 & 1.564 & 1.557 & 1.564 & 1.568 \\
\hline \multirow{3}{*}{ 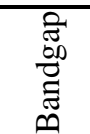 } & $\mathrm{k} / \mathrm{a}$ & -0.81 & -0.79 & -0.94 & -0.79 & -0.81 \\
\hline & $\mathrm{k} / \mathrm{b}$ & -0.811 & -0.947 & -1.288 & -0.947 & -0.811 \\
\hline & $\mathrm{k} / \mathrm{c}$ & -0.811 & -0.812 & -0.814 & -0.812 & -0.811 \\
\hline
\end{tabular}

Table II: Rgies for LDA-X, k/a, k/b and k/c

\begin{tabular}{|c|c|c|c|c|c|c|}
\hline \multirow{3}{*}{ 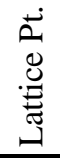 } & $\mathrm{k} / \mathrm{c}$ & 0 & 0.25 & 0.5 & 0.75 & 1 \\
\hline & $\mathrm{k} / \mathrm{b}$ & 0 & 0.25 & 0.5 & 0.75 & 1 \\
\hline & $\mathrm{k} / \mathrm{c}$ & 0 & 0.25 & 0.5 & 0.75 & 1 \\
\hline \multirow{3}{*}{ 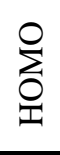 } & $\mathrm{k} / \mathrm{c}$ & -1.42 & -1.43 & -1.43 & -1.43 & -1.42 \\
\hline & $\mathrm{k} / \mathrm{b}$ & -1.42 & -1.44 & -1.5 & -1.44 & -1.42 \\
\hline & $\mathrm{k} / \mathrm{c}$ & -1.42 & -1.43 & -1.43 & -1.43 & -1.42 \\
\hline \multirow{3}{*}{ 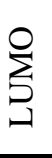 } & $\mathrm{k} / \mathrm{c}$ & -0.34 & -0.35 & -0.36 & -0.35 & -0.34 \\
\hline & $\mathrm{k} / \mathrm{b}$ & -0.34 & -0.31 & -0.25 & -0.31 & -0.34 \\
\hline & $\mathrm{k} / \mathrm{c}$ & -0.34 & -0.35 & -0.36 & -0.35 & -0.34 \\
\hline \multirow{3}{*}{ 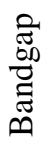 } & $\mathrm{k} / \mathrm{c}$ & -1.08 & -1.08 & -1.07 & -1.08 & -1.08 \\
\hline & $\mathrm{k} / \mathrm{b}$ & -1.08 & -1.13 & -1.25 & -1.13 & -1.08 \\
\hline & $\mathrm{k} / \mathrm{c}$ & -1.08 & -1.08 & -1.07 & -1.08 & -1.08 \\
\hline
\end{tabular}

Table III: Electron energies for PWC91, k/a, k/b and k/c

\begin{tabular}{|c|c|c|c|c|c|c|}
\hline \multirow{3}{*}{ 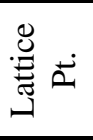 } & $\mathrm{k} / \mathrm{a}$ & 0 & 0.2 & 0.6 & 0.8 & 1 \\
\hline & $\mathrm{k} / \mathrm{b}$ & 0 & 0.29 & 0.57 & 0.71 & 1 \\
\hline & $\mathrm{k} / \mathrm{c}$ & 0 & 0.25 & 0.5 & 0.75 & 1 \\
\hline \multirow{3}{*}{$\sum_{\substack{0 \\
\hdashline}}^{0}$} & $\mathrm{k} / \mathrm{a}$ & -1.68 & -1.65 & -1.62 & -1.65 & -1.68 \\
\hline & $\mathrm{k} / \mathrm{b}$ & -1.68 & -1.69 & -1.72 & -1.69 & -1.68 \\
\hline & $\mathrm{k} / \mathrm{c}$ & -1.68 & -1.68 & -1.69 & -1.68 & -1.68 \\
\hline \multirow{3}{*}{ 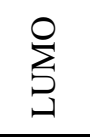 } & $\mathrm{k} / \mathrm{a}$ & -0.56 & -0.57 & -0.58 & -0.57 & -0.56 \\
\hline & $\mathrm{k} / \mathrm{b}$ & -0.56 & -0.53 & -0.51 & -0.53 & -0.56 \\
\hline & $\mathrm{k} / \mathrm{c}$ & -0.56 & -0.57 & -0.58 & -0.57 & -0.56 \\
\hline \multirow{3}{*}{ 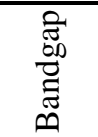 } & $\mathrm{k} / \mathrm{c}$ & -1.12 & -1.08 & -1.04 & -1.08 & -1.12 \\
\hline & $\mathrm{k} / \mathrm{b}$ & -1.12 & -1.16 & -1.21 & -1.16 & -1.12 \\
\hline & $\mathrm{k} / \mathrm{c}$ & -1.12 & -1.11 & -1.11 & -1.11 & -1.12 \\
\hline
\end{tabular}




\section{A. LDA-X Results}

As the electron's spin, this density function defines the spin density of freedom; spin can be spin up or spin down. Although local density function was derived, homogenous electron gas is sufficiently accurate for homogenous cases as well. The local density function is good with the error cancellation, and the spherical average is well approximated [16].

\section{B. PBE Results}

PBE is a short form of Perdew-BurkeErnzerhof exchange and correlation functional. PBE is a simplification of PW91, which yields almost identical numerical results with simpler formulas from a simpler derivation [18]. Calculation produced using PBE is shown below in Table II.

\section{PWC91 Results}

PW is the Perdew-Wang correlation functional. The difference in calculation with PBE and PW91 is small. PW91 is more complex and involves more gradients in it, while PBE is a simplified version of PW91 [17].

\section{Conclusions}

Pentacene is a flexible material and has $\pi$ conjugate [14]. The electronic conductivity in Pentacene opens a new chapter for the semiconductor industry [6], as Pentacene is a simple and basic organic polymer [7]. The results shown in the graphical form have a dot and continuous line in them. The continuous lines represent energies in valance and conduction band. Results consistently show that when the conduction band goes in minima at the same point valance bond goes for its maxima. This showed that Pentacene is a material with a direct bandgap. As described earlier in this chapter that direct bandgaps are good for the optical properties and materials with direct bandgaps produce bright visible lights. Hence Pentacene is the material that is a good candidate for material used for organic light-emitting diode.

The other important thing to note is that the bandgap calculated by these methods is in the range of -0.8 to $-1.2 \mathrm{eV}$ [4]. This means that Pentacene posses' good semiconductor and transistors made of this semiconductor will have good switching time. Hence Pentacene is not a bad choice for the manufacturing of organic fieldeffect transistors.

\section{APPendix PARAMeTer ANd Cell Code}

* param files normally contain following lines it in it

comment: Diamond Geometry Optimization task : GeometryOptimization xc_functional : PW91 opt_strategy: Speed basis_precision: Precise fix_occupancy : true metals_method : dm geom_method: BFGS

*.cell files have the following lines in it.

\%BLOCK LATTICE_ABC

2.4962382 .4962382 .496238

60.060 .060 .0

$\%$ ENDBLOCK LATTICE_ABC

$\%$ BLOCK POSITIONS_FRAC

$\mathrm{C} 0.0000 .0000 .000$

C 0.2500 .2500 .250

$\%$ ENDBLOCK

POSITIONS_FRAC

Kpoints_MP_grid 222

SYMMETRY_GENERATE

\%BLOCK SPECIES_POT

$\mathrm{Cd}$ : castep\PseudopotentialsMS\C

_00.recpot

\%ENDBLOCK SPECIES_POT 


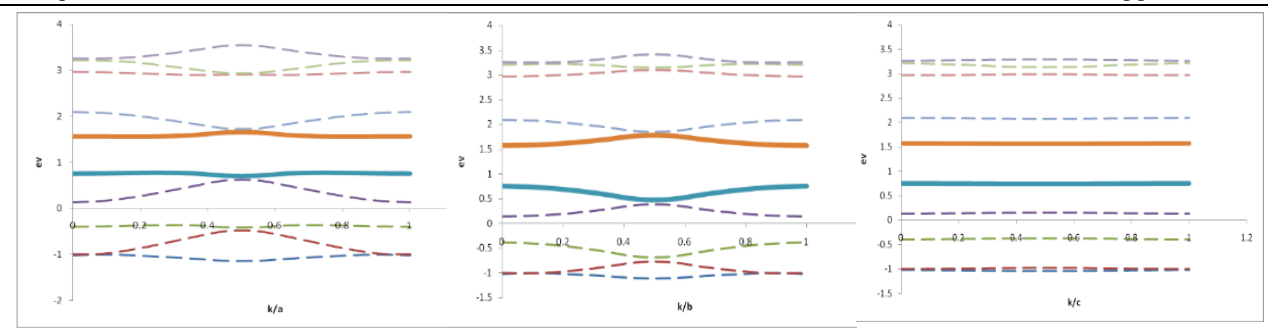

Fig 4. Electron Energies For LDA-X: k/a, k/b and k/c

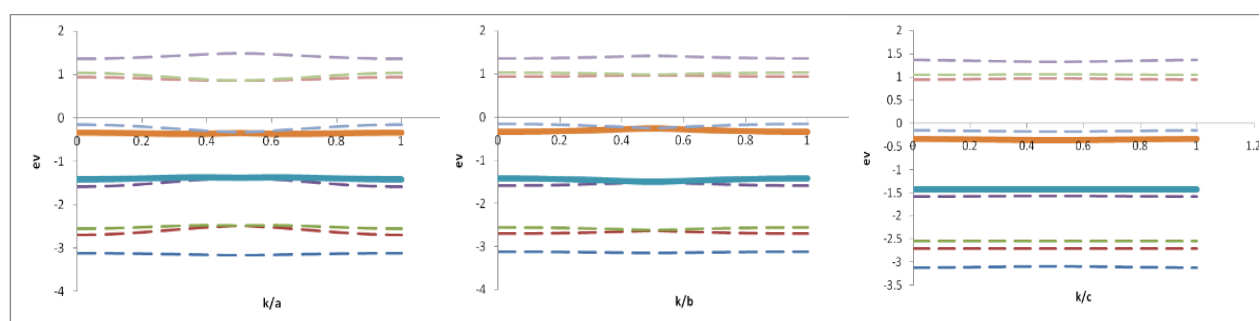

Fig 5. Electron Energies For PBE: k/a, k/b and k/c

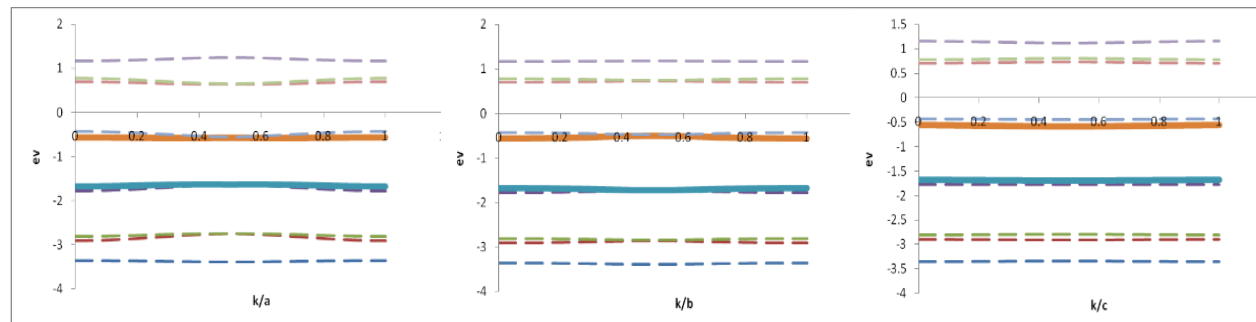

Fig 6. Electron energies for PW91: k/a, k/b and k/c

\section{CONFLICT OF INTEREST}

The authors declare no conflict of interest.

\section{Author ConTributions}

Nasif Raza Jaffri and Shi designed the developed the research idea. All the other others helped in in execution of simulations and collection of results.

\section{ACKNOWLEDGMENT}

The authors wish to extend their gratitude to NSFC for sponsoring the research (No 61571189, 61871181) and to the State Administration of Foreign Experts Affairs for supporting the 111 projects (ref: B13009)

\section{References}

[1] C. $\mathrm{Lu}$ et al., "Progress in flexible organic thin-film transistors and integrated circuits," Sci. Bull., vol. 61, no. 14, pp. 1081-1096, Jul. 2016.

[2] K. Takagi, T. Kuroda, M. Sakaida, and H. Masu, "Molecular design for tuning electronic structure of $\pi$-conjugated polymers containing fused dithienobenzimidazole units," Polymer (Guildf)., vol. 107, pp. 191-199, Dec. 2016.

[3] P. Petelenz, Z. G. Soos, and E. Umbach, "Electronic processes in organic solids," Chem. Phys., vol. 325, no. 1, pp. 1-2, Jun. 2006.

[4] N. T. Kalyani, H. Swart, and S. J. Dhoble, "Luminescence in Organic Semiconductors," in Principles and 
Applications of Organic Light Emitting Diodes (OLEDs), Elsevier, 2017, pp. 39-64.

[5] Y. Shirota and H. Kageyama, "Organic materials for optoelectronic applications: Overview," in Handbook of Organic Materials for Electronic and Photonic Devices, Elsevier, 2019, pp. 3-42.

[6] D. Schlettwein, "Electronic Properties of Molecular Organic Semiconductor Thin Films," in Supramolecular Photosensitive and Electroactive Materials, Elsevier, 2001, pp. 211-338.

[7] M. Nitani, K. Nakayama, K. Maeda, M. Omori, and M. Uno, "Organic temperature sensors based on conductive polymers patterned by a selective-wetting method," Org. Electron., vol. 71, pp. 164-168, Aug. 2019.

[8] F. Zhang, M. Funahashi, and N. Tamaoki, "Flexible field-effect transistors from a liquid crystalline semiconductor by solution processes," Org. Electron., vol. 11, no. 3, pp. 363 368, Mar. 2010.

[9] G. Casula et al., "A flexible organic memory device with a clearly disclosed resistive switching mechanism," Org. Electron., vol. 64, pp. 209-215, Jan. 2019.

[10] G. Fortunato, A. Pecora, and L. Maiolo, "Polysilicon thin-film transistors on polymer substrates," Mater. Sci. Semicond. Process., vol. 15, no. 6, pp. 627-641, Dec. 2012.

[11] K. Ryu, I. Kymissis, V. Bulovic, and C. G. Sodini, "Direct extraction of mobility in pentacene OFETs using C$\mathrm{V}$ and I-V measurements," IEEE Electron Device Lett., vol. 26, no. 10, pp. 716-718, Oct. 2005.

[12] V. Coropceanu, J. Cornil, D. A. da Silva Filho, Y. Olivier, R. Silbey, and J.-L. Brédas, "Charge Transport in Organic Semiconductors," Chem. Rev., vol. 107, no. 4, pp. 926-952, Apr. 2007.

[13] P. F. Van Hutten and G. K. Sujan, "Organic Conductors and Semiconductors, Structure and
Morphology of," in Reference Module in Materials Science and Materials Engineering, Elsevier, 2016.

[14] T. Sekitani et al., "Bending experiment on pentacene field-effect transistors on plastic films," Appl. Phys. Lett., vol. 86, no. 7, p. 073511, 2005.

[15]F.-C. Chen, "Organic Semiconductors," in Encyclopedia of Modern Optics, Elsevier, 2018, pp. 220-231.

[16]M. Ribeiro, "Electronic band gaps corrections using total energy with DFT/LDA-1/2 quasi-particle approximation," Comput. Mater. Sci., vol. 167, pp. 228-236, Sep. 2019.

[17] J. Bergès, J. Caillet, J. Langlet, and J. Kozelka, "Hydration and inverse hydration' of platinum(II) complexes: an analysis using the density functionals PW91 and BLYP," Chem. Phys. Lett., vol. 344, no. 5-6, pp. 573577, Aug. 2001.

[18] I. A. Pašti, A. Jovanović, A. S. Dobrota, S. V. Mentus, B. Johansson, and N. V. Skorodumova, "Atomic adsorption on pristine graphene along the Periodic Table of Elements - From PBE to non-local functionals," Appl. Surf. Sci., vol. 436, pp. 433-440, Apr. 2018. 